\title{
Reutilización de vidrio reciclado y residuos cerámicos en la obtención de gres porcelánico. Eco-logik
}

\author{
V. R. TRILLES-LÁZARO', S. ALLEPUZ ${ }^{2}$ \\ 'Director Técnico de Azulejos Plaza \\ ${ }^{2}$ Jefe de I+D Camacho Recycling
}

Este Sistema ha recibido un premio Alfa de Oro en la Feria Internacional CEVISAMA VALENCIA 2011

\begin{abstract}
Plaza Cerámicas ha llevado a cabo, en colaboración con el Instituto de Tecnología Cerámica (ITC) de la Universidad Jaume I de Castellón, un proyecto de investigación orientado a potenciar el uso de vidrio reciclado y residuos procedentes del proceso de producción cerámico en la fabricación industrial de baldosas cerámicas. El resultado de este estudio es 'ECO-LOGIK', una baldosa de gres porcelánico fabricada a partir de materias primas con un alto contenido en materiales procedentes del reciclaje-más de un $85 \%$ en el soporte de la pieza-, lo que la convierte en un producto de alta calidad, sostenible y respetuoso con el medio ambiente.
\end{abstract}

Palabras clave. Ecologic, recycled glass, porcelain tiles

Porcelanic tiles obtained by use of recycled glass and other ceramic residues. Eco-logik

Plaza Ceramicas has carried out, in collaboration with the ITC (Instituto de Technologica Ceramica) from the Jaume I University of Castellon, a project investigating the increased usage of recycled glass and residues resulting from the manufacturing process of ceramic tiles. The result of this study is "Eco-Logik", a porcelain tile produced from raw materials with a high content of recycled materials - more than an $85 \%$ of the tile itself - which makes it a high quality product, in accordance with the welfare of the Environment.

Key words: Ecologic, recycled glass, porcelain tiles

La utilización de productos procedentes del reciclado de otros productos cerámicos es una de las vías mas relevantes utilizados por la industria cerámica para la reducción de costes y el establecimiento de una modelo de producción más sostenible. El abanico de residuos empleados y la mayor calidad de los productos a cuya producción se aplican, es una de las constantes de la industria cerámica

Plaza Cerámicas ha llevado a cabo, en colaboración con el Instituto de Tecnología Cerámica (ITC) de la Universidad Jaume I de Castellón, un proyecto de investigación orientado a potenciar el uso de vidrio reciclado y residuos procedentes del proceso de producción cerámico en la fabricación industrial de baldosas cerámicas. El resultado de este estudio es 'ECOLOGIK', una baldosa de gres porcelánico fabricada a partir de materias primas con un alto contenido en materiales procedentes del reciclaje -más de un $85 \%$ en el soporte de la pieza-, lo que la convierte en un producto de alta calidad, sostenible y respetuoso con el medio ambiente.

Plaza Cerámicas, es una empresa azulejera con casi medio siglo de trayectoria ubicada en la Alcora (Castellón), comercializará próximamente una baldosa de estas carácteristica:'ECO-LOGIK', nacida de un proyecto de investigación que ha llevado a cabo en colaboración con la Universidad Jaume I. El resultado más importante de este estudio es el desarrollo de un sistema productivo que permite reutilizar el vidrio procedente del uso industrial y doméstico como materia prima para la fabricación de baldosas cerámicas. El estudio determinó que el vidrio, después de ser clasificado en función de su composición y su distribución granulométrica, puede ser tratado y posteriormente incorporado a las distintas partes que conforman una baldosa cerámica (soporte, engobe y esmalte), logrando unas prestaciones técnicas idénticas a las del gres porcelánico convencional.
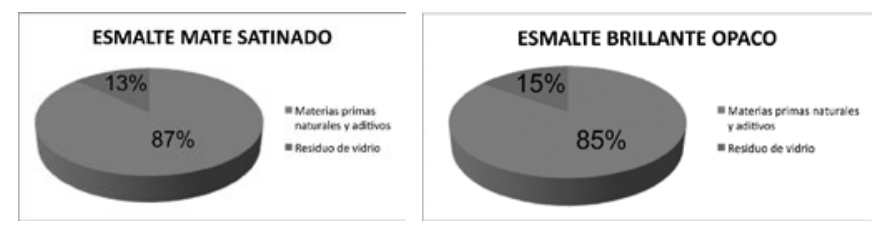

Figura 1: Cantidad de vidrio reciclado en dos esmaltes Eco-logik. 
Otra novedad importante que presenta este sistema es que permite asimismo la incorporación de chamotas ( material cocido desechado, procedente de la fabricación del gres porcelánico) a las composiciones de la pasta de gres porcelánico despúes de acondicionar su granulometría. Así, se han obtenido soportes cerámicos de alta gresificación a partir de la introducción de vidrio reciclado, chamota y lodos, elevando hasta más de un $85 \%$ el porcentaje de materiales reciclados utilizados en su fabricación. El estudio ha permitido también formular composiciones de esmaltes en base a la introducción del vidrio de recuperación, tanto de naturaleza sódico-cálcica como de naturaleza borosilicatada.

\section{GRADO DE REUTILIZACIÓN DE RECURSOS}

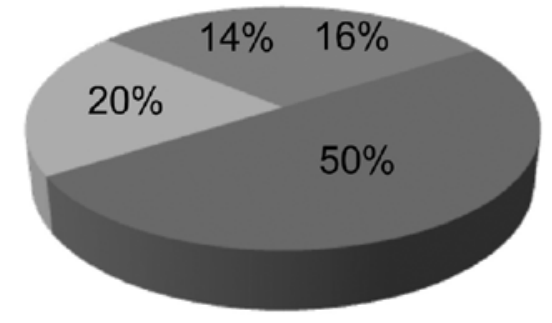

E Reducción de materias

primas

"neducción de aguas

limpias

" Reducción de energía

= Recursos no reutilizables

Figura 2: Grado de reutilización de recursos en la baldosa Eco-logik.

Actualmente ya son muchas las empresas que, en mayor o menor medida, reciclan diversos residuos derivados del proceso productivo cerámico, como los lodos procedentes de las balsas de decantación, las aguas sucias procedentes del lavado o los tiestos crudos. Añadir a estos materiales el vidrio -un material ajeno al proceso productivo cerámico- y de chamotas, procedentes de piezas de desecho, supone un paso adelante en el esfuerzo por convertir la fabricación industrial de cerámica de alta calidad en un ciclo sostenible y con un menor impacto en nuestro medio ambiente.

Plaza Cerámicas, fundada en 1962, ha demostrado durante casi cincuenta años de actividad empresarial un sólido compromiso con el medio ambiente y una preocupación por reducir al máximo el impacto ecológico de sus procesos industriales. Como viene haciendo desde el comienzo de su trayectoria, en los actuales tiempos de crisis económica
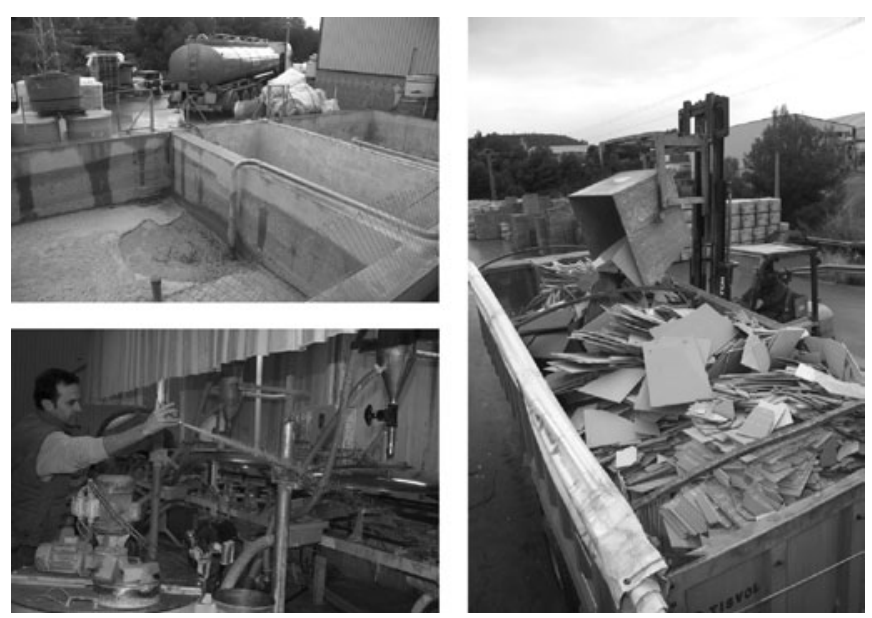

Figura 3: Distintos residuos generados en el proceso de fabricación cerámica. la empresa castellonense apuesta por el desarrollo del $\mathrm{I}+\mathrm{D}+\mathrm{i}$ como una apuesta de futuro y de responsabilidad medioambietal.

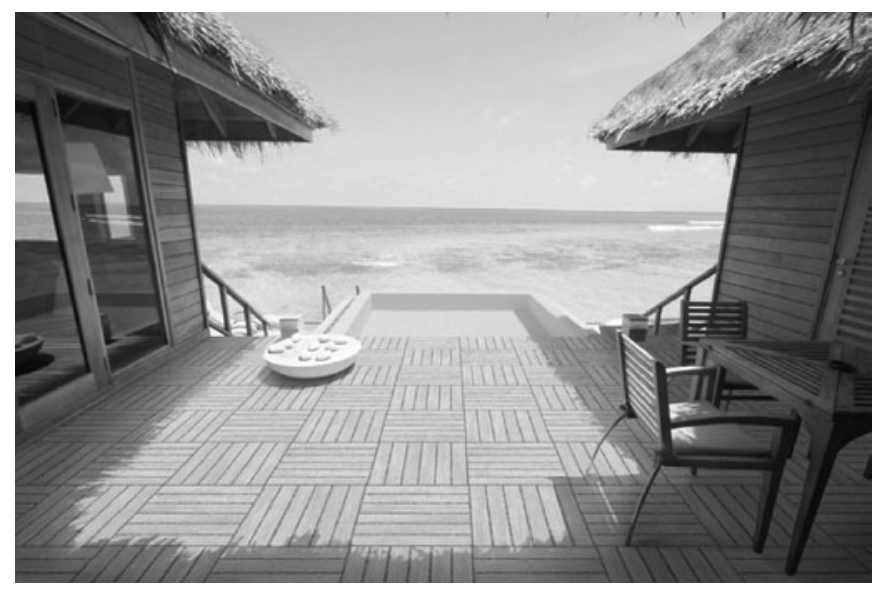

Figura 4: Modelo Garden 60 x 60.

\section{BIBLIOGRAFÍA:}

1. “"ENCICLOPEDIA CERÁMICA”, P.Escribano, J.B. Carda y E. Cordoncillo eds., Vol. I, II y III, Ed. Faenza Editrice Ibérica, Castellón, 2001.

2. Manuel Irún Molina, "Planificación Ecoeficiente de Producción. Desarrollo de Ecobaldosas Cerámicas de Gres de Pasta Roja". Tesis Doctoral. Escuela Superior de Tecnología y Ciencias Experimentales, Universitat Jaume I, Castellón, 2002.

3. M. Seoánez, "Ecología industrial: ingeniería medioambiental aplicada a la industria y a la empresa", Ed. Mundi-Prensa, Madrid, 1998.

4. Fundación Entorno, "Libro Blanco de la Gestión Medioambiental en la Industria Española”, Ed.Mundi-Prensa, Madrid, 1998.

5. J.M. Rebollo, F. Corma, "Modelo para la determinación de aspectos e impactos medioambientales en el sector cerámico", EDICERAM, 1, 19-37, (2000).

6. G. Monrós, M. Llusar, M.A. Tena, “La adaptación medioambiental de la industria cerámica", Técnica Cerámica, 283, 581-594, (2000).

7. G. Busani y C. Palmonari, "Piastrelle ceramiche \& ambiente", Ed. Assopiastrelle, Modena, 1995.

8. J.E. Enrique, E. Monfort, F. Ferrando, J.V. Agramunt, “Gestión de vertidos em la fabricación de baldosas cerámicas" Técnica Cerámica, 246, 478-485, (1996).

9. J.E. Enrique, E. Monfort, "Situación actual y perspectivas de futuro de los residuos de la industria cerámica", Cerámica Información, 221, 19-32, (1996).

10. E. Monfort, J. García-Ten, P. Velasco, M. Monzó, S. Mestre, J.C. Jarque, "Reciclado de tiesto para composiciones de pavimento y revestimiento rojo (I)", Técnica Cerámica 292, 450-458, (2001).

11. E. Monfort, J. García-Ten, P. Velasco, M. Monzó, S. Mestre, J.C. Jarque, "Reciclado de tiesto para composiciones de pavimento y revestimiento rojo (II)", Técnica Cerámica 293, 629-633, (2001).

12. S. Mestre, E. Sánchez, J. García-Ten, J. Sánchez, C. Soler, J. Portolés, J. Sales, "Utilización de la teoría de Kubelka-Munk para optimizar el reciclado de residuos crudos de gres porcelánico", Bol. Soc. Esp. Ceram.Vidr, 41(1), 429435, (2002).

13. J. García-Ten, G. Mallol, E. Bou, G. Silva, J. Fernández, A. Molina, J. Romera, "Recycling marble working wastes in manufacturing ceramic products. II Ceramic wall tile manufacture. Ceramic Forum International, DKG 80(10), 30-32, (2003).

14. A. Blasco, A. Escardino y G. Busani, "Tratamiento de emisiones gaseosas, efluentes líquidos y residuos sólidos de la industria cerámica", Edita: Instituto de Tecnología Cerámica-Asociación de Industrias Cerámicas, Castellón, 1992.

15. M. Irún, I. Nebot-Díaz y J.B. Carda, “Situación medioambiental de la fabricación de fritas y esmaltes cerámicos. Contribución de las emisiones atmosfércicas a la calidad del aire ambiente". $5^{\circ}$ Congreso Nacional de Medio Ambiente. Comunicaciones Técnicas, Madrid, 2000.

16. M. Irún, "Influencia de las variables del proceso de producción de fritas cerámicas en las emisiones atmosféricas, Tesis de Licenciatura, Departamento de Química Inorgánica y Orgánica, Universitat Jaume I, Castellón, 1999.

17. J. Peñalvert, M.D. Llanes, E. Cerisuelo, L.Sánchez-Muñoz y J.B. Carda, "Nuevas pastas para gres porcelánico de cocción roja". 40 CONGRESO NACIONAL DE LA SOCIEDAD ESPAÑOLA DE CERÁMICA Y VIDRIO, Onda (Castellón), 2000. 